\title{
Refractory Peritonitis and Small Bowel Ileus: A Case of Encapsulating Peritoneal Sclerosis Secondary to Mycobacterium abscessus Peritonitis
}

\author{
Soroush Rouhani ${ }^{1}$, Nikesh Adunuri ${ }^{2}$ \\ ${ }^{1}$ Department of Medicine, London Health Sciences Centre, Schulich School of Medicine, University of Western Ontario, London, ON, Canada \\ ${ }^{2}$ Department of General Internal Medicine, Windsor Regional Hospital, Schulich School of Medicine, University of Western Ontario, London, ON, Canada
}

Received: 07/01/2022

Accepted: $14 / 01 / 2022$

Published: 07/03/2022

\begin{abstract}
How to cite this article: Rouhani S, Adunuri N. Refractory peritonitis and small bowel ileus: a case of encapsulating peritoneal sclerosis secondary to Mycobacterium abscessus peritonitis. EJCRIM 2022;9: doi:10.12890/2022_003173.
\end{abstract}

Conflicts of Interests: The authors declare there are no competing interests.

This article is licensed under a Commons Attribution Non-Commercial 4.0 License

\section{ABSTRACT}

Introduction: Complications of peritoneal dialysis (PD) such as exit-site infections and PD peritonitis are common reasons for admission to General Internal Medicine wards. Culprit organisms range from typical skin flora to rarer complicated atypical organisms such as nontuberculous mycobacteria. Encapsulating peritoneal sclerosis (EPS) is a rarer complication of PD characterized by peritoneal inflammation, ileus and fibrosis with high morbidity, few management options, and poor prognosis.

Case Description: We report the case of a patient with a history of end-stage renal disease on peritoneal dialysis who presented with undifferentiated peritonitis and ileus refractory to standard antimicrobial therapy. Subsequent ascitic cultures were positive for Mycobacterium abscessus, and CT imaging was consistent with EPS. To date, EPS secondary to M. abscessus peritonitis has not previously been described.

Discussion and Conclusion: This report describes the diagnostic process and treatment offered to this patient and his treatment outcomes over 8 months. It highlights the importance of prompt identification of patients at risk, timely eradication of high-risk pathogens, and transition to haemodialysis to limit morbidity and improve patient prognosis.

\section{LEARNING POINTS}

- Encapsulating peritoneal sclerosis secondary to Mycobacterium abscessus peritonitis has not been described to date. M. abscessus and other non-tuberculous mycobacterial organisms should be suspected in peritoneal dialysis patients who present with refractory peritonitis and ileus.

- Encapsulating peritoneal sclerosis is an inflammatory phenomenon characterized by peritoneal inflammation, fibrosis and bowel ileus in the setting of peritoneal dialysis or recurrent infectious insults with high-risk organisms. Diagnosis depends on characteristic imaging coupled with compatible clinical symptoms.

- Treatment involves transition to haemodialysis, infection eradication, immunosuppression and anti-hormonal treatment (e.g., tamoxifen). Despite these options, prognosis remains very poor.

\section{KEYWORDS}

Encapsulating peritoneal sclerosis, peritoneal dialysis, peritonitis, non-tuberculous mycobacteria, Mycobacterium abscessus 


\section{BACKGROUND}

Peritoneal dialysis (PD) is a common and effective method of renal replacement therapy (RRT) in patients with end-stage renal disease (ESRD). Although generally well tolerated, patients are at increased risk of several infectious complications. These include exit-site infections (ESI), PD catheter tunnel infections (TI) and PD peritonitis, which are all common reasons for admission to General Internal Medicine wards. These infections are often polymicrobial, typically involving skin and enteric flora. Less frequently, fungal and mycobacterial species are involved ${ }^{[1]}$.

Although most PD-related infections are uncomplicated and resolve with appropriate antimicrobial therapy, they represent a significant source of morbidity and carry the risk of further systemic complications. Repeat infectious insults predispose patients to encapsulating peritoneal sclerosis (EPS), a rare yet serious inflammatory phenomenon that results in diffuse fibrosis and ileus, with a severe symptom burden and few robust management options. Its prevalence is estimated to be $0.4-8.9 \%{ }^{[2]}$. The pathophysiology is believed to be secondary to chronic inflammatory processes from repeat infections and from the PD process itself, with a higher risk in Staphylococcus aureus, pseudomonal and fungal infections ${ }^{[3]}$.

Much more rarely, non-tuberculous mycobacterial (NTBM) peritonitis, particularly with Mycobacterium fortuitum species, have been described in the context of EPS ${ }^{[4]}$. Other NTBM species are far less frequently identified in developing EPS. Herein, we describe the first case of EPS secondary to recurrent Mycobacterium abscessus infections, and its development over the following months.

\section{CASE DESCRIPTION}

A 48-year-old man presented to hospital with a 3-day history of fever, malaise and generalized abdominal pain. His past medical history was relevant for ESRD secondary to bilateral vesicoureteral reflux. He had a history of deceased donor renal transplant in 1990 (graft failure at 6 months), and living related donor renal transplant in 2000 (graft failure at 11 years). He was transitioned to haemodialysis from 2011 to 2017. He was transitioned to peritoneal dialysis between 2017 and 2020, which was complicated by recurrent S. aureus and M. abscessus ESI, necessitating transition back to haemodialysis. His PD catheter was removed 3 days before he presented to hospital.

On admission, he was empirically treated with broad-spectrum antibiotics (piperacillin-tazobactam) with worsening of symptoms of nausea and vomiting. His ascitic cultures were positive for $\mathrm{M}$. abscessus complex. CT imaging revealed small volume ascites with no perforation, mild bowel wall dilation $(2.7 \mathrm{~cm})$, and extensive omental and peritoneal linear calcifications (Fig. 1). The overall constellation of findings was consistent with a diagnosis of encapsulating sclerosing peritonitis.
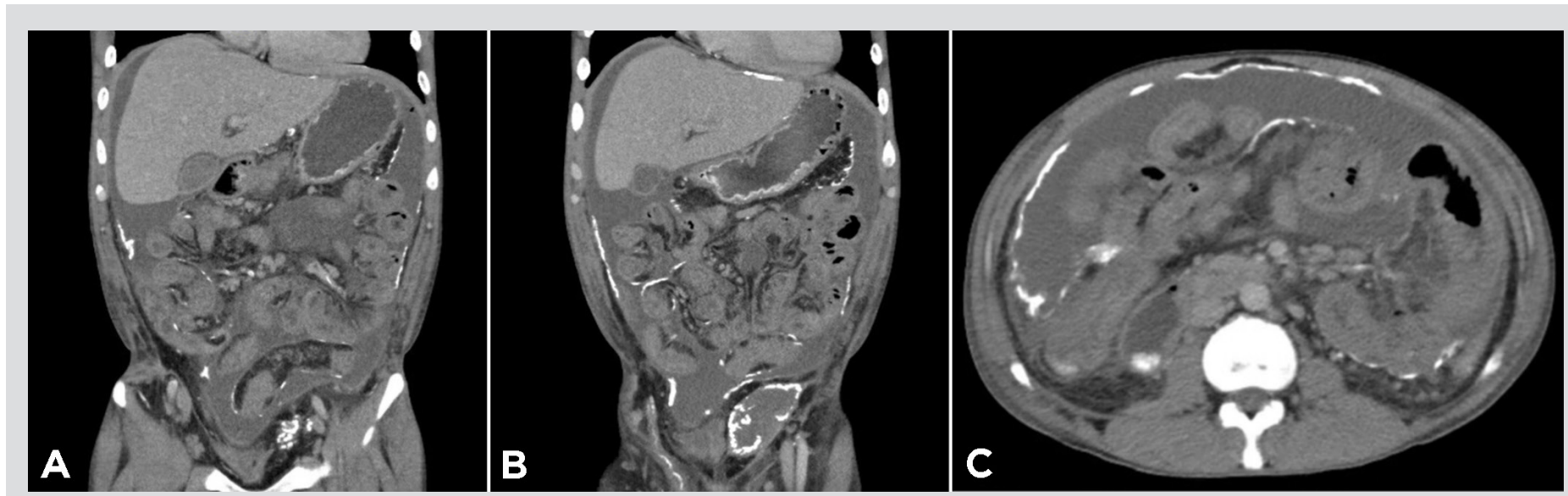

Figure 1. Computed tomographic topogram (A) and computed tomographic scan of the chest with contrast (B) showing the presence of air and a fluid component (indicated by a blue arrow) in the pericardial space

His treatment was changed to amikacin, azithromycin, cefoxitin, linezolid and prednisone $25 \mathrm{mg}$ daily. His culture sensitivity findings revealed resistance to ciprofloxacin, macrolides and linezolid, with intermediate resistance to cefoxitin, imipenem and tigecycline. Subsequently, his azithromycin and linezolid were replaced with intravenous tigecycline. His symptoms improved over the following days with active outpatient infectious disease follow-up.

At his last follow-up a few months ago, the patient was doing well, with resolution of his fever, abdominal pain, nausea and vomiting. He did not have any refractory obstructive symptoms. He will continue his treatment regimen for an intended total duration of 12 months. 


\section{DISCUSSION}

EPS is a rare but serious phenomenon that most commonly occurs as a complication of peritoneal dialysis. The pathophysiology is described as an inflammatory process resulting in diffuse fibrocollagenous deposition along the small intestine, with subsequent thickening of the peritoneum ${ }^{[5]}$. EPS is initially asymptomatic as thickening progresses (encapsulating stage) until a critical degree of thickness is achieved and obstructive symptoms develop (ileus stage) ${ }^{[2]}$. To date, there is no consensus on diagnostic criteria, and diagnosis of EPS relies on radiological findings of diffuse peritoneal calcification, bowel dilatation and tethering, in conjunction with symptoms of ileus. In certain cases, EPS may be heralded by an inflammatory state including fever, malaise and elevated serum inflammatory markers ${ }^{[2]}$. Consequently, the incidence of EPS among PD patients is poorly described, and is further complicated by delayed diagnosis and often vague symptomatology ${ }^{[2,3]}$.

EPS is not exclusive to PD, and many secondary causes have been identified, including bacterial and mycobacterial peritonitis, among others ${ }^{[5]}$. Approximately $3 \%$ of PD-associated infections are attributed to NTBM, with M. abscessus only representing $8.8 \%$ of these cases in published case series ${ }^{[6]}$. From our literature search, EPS secondary to M. abscessus peritonitis, with or without concomitant PD, has not been described to date.

The treatment of EPS is based on the underlying aetiology. PD patients are often transitioned to haemodialysis in an attempt to halt the progression of fibrosis. Certain therapies including corticosteroids, tamoxifen and immunosuppression are offered, although data on the efficacy of these therapies remain inconclusive ${ }^{[7,8]}$. In refractory cases, surgical treatment may be sought ${ }^{[7]}$. Any underlying infectious process is treated with the goal of confirming microbial clearance with repeat fluid culture.

M. abscessus can be difficult to treat due to inherent multi-drug resistance to macrolides, aminoglycosides, rifamycins, tetracyclines and B-lactams via multiple mechanisms ${ }^{[9]}$.

Unfortunately, our patient had already discontinued PD by the time of admission with minimal ascitic fluid remaining after one diagnostic paracentesis, thus precluding repeat ascitic culture to ensure clearance and complicating antimicrobial management. Long-term therapy is also complicated by the significant toxicity associated with available antimicrobials, which highlights the importance of capturing such patients early, prior to PD catheter removal, in order to obtain repeat fluid cultures more easily. This case also highlights the importance of recognizing NTBM as a possible culprit in patients with suspected PD peritonitis who are not improving clinically on standard empiric therapy. Symptoms such as anorexia, nausea, vomiting and weight loss should raise suspicion of underlying EPS. Given PD patients' risk of mycobacterial peritonitis, as well as the independent association of mycobacterial infections with EPS (outside the context of PD), mycobacterial cultures should be sent early in these cases. Furthermore, consideration should be given to early CT imaging in patients with PD-associated infection secondary to NTBM (including M. abscessus), to detect radiological findings associated with EPS and guide earlier diagnosis, with the aim of limiting progression, and possible avoidance of medical/surgical intervention.

\section{REFERENCES}

1. Washida N, Itoh H. The role of non-tuberculous mycobacteria in peritoneal dialysis-related infections: a literature review. Contrib Nephrol $2018 ; 196: 155-61$.

2. Brown AB, Bargman J, Biesen W, Chang M, Finkelstein F, Hurst H, et al. Length of time on peritoneal dialysis and encapsulating peritoneal sclerosis - position paper for ISPD: 2017 update. Perit Dial Int 2017;37(4):362.

3. Kawaguchi Y, Kawanishi H, Mujais S, Topley N, Oreopoulos DG. Encapsulating peritoneal sclerosis: definition, etiology, diagnosis, and treatment. International Society for Peritoneal Dialysis Ad Hoc Committee on Ultrafiltration Management in Peritoneal Dialysis. Peri Dial Int 2000;20(Suppl 4):S43-55.

4. Simbli MA, Niaz FA, AI-Wakeel JS. Encapsulating peritoneal sclerosis in a peritoneal dialysis patient presenting with complicated Mycobacterium fortuitum peritonitis. Saudi J Kidney Dis Transpl 2012;23(3):635-41.

5. Danford CJ, Lin SC, Smith MP, Wolf JL. Encapsulating peritoneal sclerosis. World J Gastroenterol 2018;24(28):3101-11.

6. Song Y, Wu J, Yan H, Chen J. Peritoneal dialysis-associated nontuberculous mycobacterium peritonitis: a systematic review of reported cases. Nephrol Dial Transplant 2012;27:1639-44.

7. Dobbie JW. Pathogenesis of peritoneal fibrosing syndromes (sclerosing peritonitis) in peritoneal dialysis. Perit Dial Int 1992;12(1):14.

8. Balasubramaniam G, Brown EA, Davenport A, Cairns H, Cooper B, Fan SLS, et al. Clinical course and management of encapsulating peritoneal sclerosis: a multicentre retrospective survey from the UK. Nephrol Dial Transplant 2009;24:3209-15.

9. Johansen MD, Herrman J, Kremer L. Non-tuberculous mycobacteria and the rise of Mycobacterium abscessus. Nat Rev Microbiol 2020;18:392-407. 